\title{
Comparison of Negative Moment Estimator with Maximum Likelihood Estimator of Inverse Rayleigh Distribution
}

Muhammad Mohsin

Department of Mathematical Sciences

COMSATS Institute of Information Technology, Lahore

Muhammad Qaiser Shahbaz

Department of Statistics, Govt. College University, Lahore

\begin{abstract}
In this paper, method of negative moments has been used to estimate the parameter of Inverse Rayleigh Distribution. The asymptotic variance to the parameter estimates has been obtained. A comparison have been made between the variance of Maximum Likelihood Estimate and the variance of negative moment of first order for Inverse Rayleigh Distribution.
\end{abstract}

Keywords: Reliability, Negative Moment, Maximum Likelihood Estimator.

\section{Introduction}

The Inverse Rayleigh Distribution was introduced in literature by Trayer (1964). In reliability and survival studies many life distributions are characterized by monotonic failure rate. The one-parameter Inverse Rayleigh Distribution with probability density function.

$$
f(x ; \theta)=\frac{2 \theta}{x^{3}} \exp \left(-\frac{\theta}{x^{2}}\right), \quad \theta>, x>0
$$

has been used as a failure time distribution in reliability study. Iliescu and Voda (1972) have been studies some properties of the Inverse Rayleigh Distribution. Also Voda (1972) discussed some properties of Maximum Likelihood Estimator of the Parameter $\theta$. The variance and the higher order moments of this distribution do not exist.

\section{Estimation of parameters}

In this section method of maximum likelihood and method of negative moments have been used for estimation of parameters for inverse Rayleigh distribution. The variance of negative moments estimator has been compared with that of maximum likelihood estimator.

\section{a) Method of Maximum Likelihood}

The log likelihood function of (1.1) is

$$
\log \mathrm{L}=\mathrm{n} \log (2 \theta)-\log \left(\prod_{\mathrm{i}=1}^{n} \mathrm{x}_{\mathrm{i}}^{3}\right)-\theta \sum_{i=1}^{n} \frac{1}{x_{i}^{2}}
$$


Differentiating (2.1) with respect to $\theta$ and equating to zero, we get

$$
\begin{array}{r}
\hat{\theta}=\frac{n}{\sum_{i=1}^{n} \frac{1}{x_{i}^{2}}} \\
\text { and } \quad \operatorname{Var}(\hat{\theta})=\frac{\theta^{2}}{n}
\end{array}
$$

From (2.2) it is clear that MLE of $\theta$ is harmonic mean of the sequences of observations.

\section{b) Method of Negative Moment}

The first order negative moment of inverse Rayleigh distribution is given by

$$
u_{(-1)}^{\prime}=\theta^{-1 / 2} \Gamma(3 / 2)
$$

The first order negative moment estimator of $\theta$ is as

$$
m_{(-1)}^{\prime}=\theta^{-1 / 2} \Gamma(3 / 2)
$$

Now partially differentiating (2.4) with respect to $\theta$, squaring both sides and taking expectation, we get the asymptotic variance of $\hat{\theta}$ as:

$$
\begin{aligned}
& E\left(\partial m_{(-1)}^{\prime}\right)^{2}=\left[-\frac{1}{2} \hat{\theta}^{-\frac{3}{2}} \Gamma(3 / 2)\right]_{\hat{\theta} \rightarrow \theta}^{2} E(\partial \hat{\theta})^{2} \\
& \operatorname{Var}\left(m_{(-1)}^{\prime}\right)=\left[\frac{1}{2} \frac{1}{\theta^{3 / 2}} \cdot \frac{\sqrt{\pi}}{2}\right]^{2} \operatorname{Var}(\hat{\theta}) \\
& \operatorname{Var}(\hat{\theta})=\left[\frac{\sqrt{\pi}}{4} \cdot \frac{1}{\theta^{3 / 2}}\right]^{-2} \operatorname{Var}\left(m_{(-1)}^{\prime}\right)
\end{aligned}
$$

To obtain the $\operatorname{Var}\left(m_{(-1)}^{\prime}\right)$, we have following result:

$$
\operatorname{Var}\left(m_{(-1)}^{\prime}\right)=\frac{1}{n} \operatorname{Var}\left(\frac{1}{x}\right)
$$

Where $\quad \operatorname{Var}\left(\frac{1}{x}\right)=\frac{1}{\theta}\left[1-\frac{\pi}{4}\right]$

Using (2.7) in (2.6), we get

$$
\operatorname{Var}\left(m_{(-1)}^{\prime}\right)=\frac{1}{\mathrm{n} \theta}\left(1-\frac{\pi}{4}\right)
$$

From (2.5), we obtain the following result.

$$
\operatorname{Var}(\hat{\theta})=\frac{4 \theta^{2}}{\mathrm{n}} \cdot\left[\frac{4}{\pi}-1\right]
$$

for large $\theta$. 
Table: $\operatorname{Var}(\hat{\theta})$ and $\operatorname{Var}(M L E)$ of Inverse Rayleigh distribution for $\mathbf{n}=10$ and $\mathbf{n}=\mathbf{3 0}$

\begin{tabular}{|c|c|c|c|c|}
\hline$\theta$ & $\operatorname{Var}(\hat{\theta}) \mathrm{n}=10$ & $\operatorname{Var}(\mathrm{MLE}) \mathrm{n}=10$ & $\operatorname{Var}(\hat{\theta}) \mathrm{n}=30$ & $\operatorname{Var}(\mathrm{MLE}) \mathrm{n}=30$ \\
\hline 0.5 & 0.0273 & 0.0250 & 0.0091 & 0.0083 \\
1.0 & 0.1091 & 0.1000 & 0.0364 & 0.0333 \\
1.5 & 0.2455 & 0.2250 & 0.0818 & 0.0750 \\
2.0 & 0.4364 & 0.4000 & 0.1455 & 0.1333 \\
2.5 & 0.6818 & 0.6250 & 0.2273 & 0.2083 \\
3.0 & 0.9818 & 0.9000 & 0.3273 & 0.3000 \\
3.5 & 1.3364 & 1.2250 & 0.4455 & 0.4083 \\
4.0 & 1.7455 & 1.6000 & 0.5818 & 0.5333 \\
4.5 & 2.2091 & 2.0250 & 0.7364 & 0.6750 \\
5.0 & 2.7273 & 2.5000 & 0.9091 & 0.8333 \\
5.5 & 3.3000 & 3.0250 & 1.1000 & 1.0083 \\
6.0 & 3.9273 & 3.6000 & 1.3091 & 1.2000 \\
6.5 & 4.6091 & 4.2250 & 1.5364 & 1.4083 \\
7.0 & 5.3455 & 4.9000 & 1.7818 & 1.6333 \\
7.5 & 6.1364 & 5.6250 & 2.0455 & 1.8750 \\
8.0 & 6.9818 & 6.4000 & 2.3273 & 2.1333 \\
8.5 & 7.8818 & 7.2250 & 2.6273 & 2.4083 \\
9.0 & 8.8364 & 8.1000 & 2.9455 & 2.7000 \\
9.5 & 9.8455 & 9.0250 & 3.2818 & 3.0083 \\
10.0 & 10.9091 & 10.0000 & 3.6364 & 3.3333 \\
\hline
\end{tabular}

Fig. 1: Comparison of $\operatorname{Var}(\hat{\theta})$ and $\operatorname{Var}(\mathrm{MLE})$ of Inverse Rayleigh Distribution for $n=10$

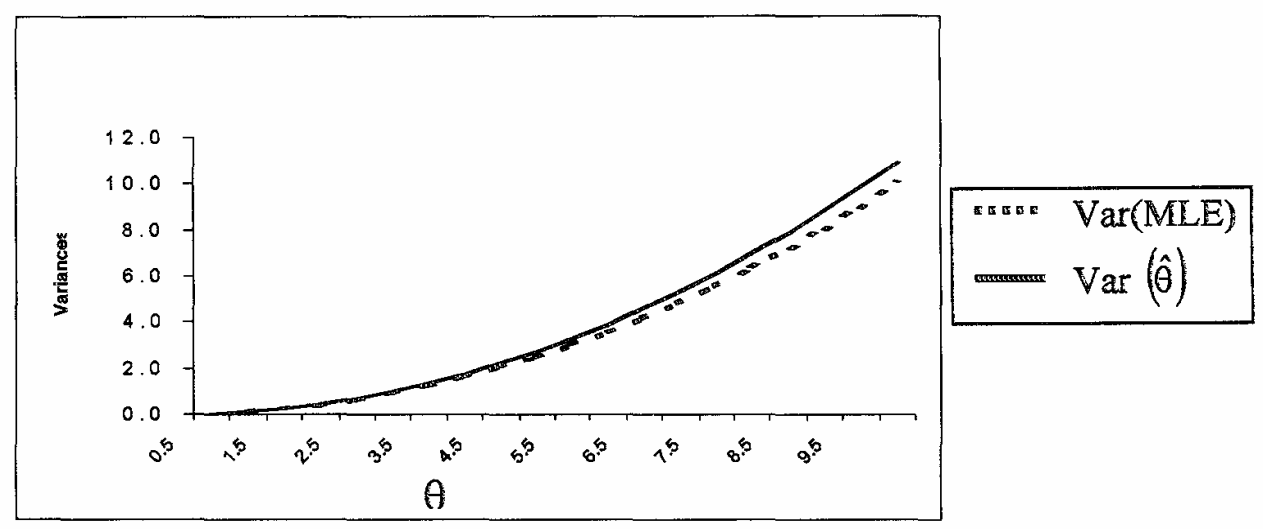


Fig. 2: Comparison of $\operatorname{Var}(\hat{\theta})$ and $\operatorname{Var}(M L E)$ of Inverse Rayleigh Distribution for $\mathbf{n}=\mathbf{3 0}$

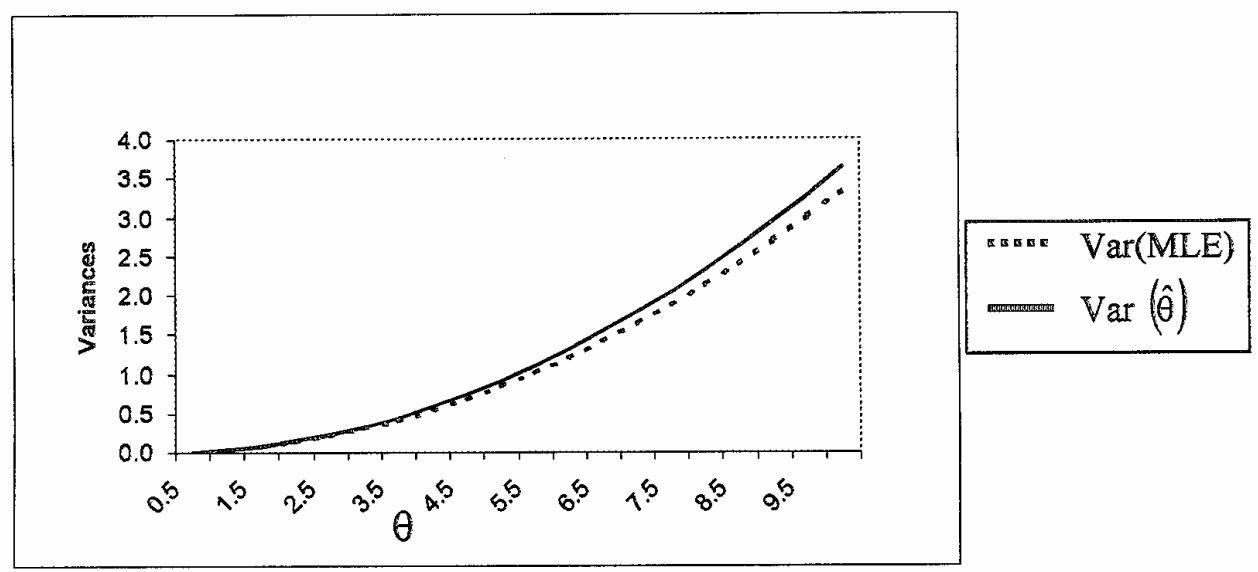

The variance of maximum likelihood estimator has been compared with the variance of negative moment estimator by graph. It is observed that the variance of the maximum likelihood estimator is efficient than the variance of negative moment estimator in case of inverse Rayleigh distribution.

\section{References}

1. Gharraph, M.K. (1993). Comparison of Estimators of Location Measures of an Inverse Rayleigh Distribution. The Egyptian Statistical Journal. 37, 295-309.

2. Mukarjee, S.P. and Maitim, S.S. (1996). A Percentile Estimator of the Inverse Rayleigh Parameter. IAPQR Transactions, 21, 63-65.

3. Voda, V. Gh. (1972). On the Inverse Rayleigh Random Variable, Pep. Statist. App. Res., JUSE, 19, 13-21.

4. $\quad$ Treyer, V. N. (1964). Doklady Acad, Nauk, Belorus, U.S.S.R.

5. Iliescu, D.V. and Voda, V. Gh. (1972). A study concerning the Inverse Rayleigh Variate, (Unpublished Manuscript). 